\title{
Chest Radiography
}

National Cancer Institute

\section{Source}

National Cancer Institute. Chest Radiography. NCI Thesaurus. Code C38103.

An $\mathrm{x}$-ray examination of the chest. 